\title{
Teachers as Assessors: A Case Study of the Gauteng Province (Republic of South Africa)
}

\author{
Tebogo Mogashoa \\ College of Education, University of South Africa \\ PO Box 392, 0003 Unisa, South Africa \\ Email: mogasti@unisa.ac.za
}

Doi:10.5901/mjss.2013.v4n14p371

\begin{abstract}
The aim of the research was to establish how assessment was implemented by teachers in selected schools in Gauteng Province. This study was underpinned by a critical discourse analysis theory in which the researcher expressed his comments or judgements based on written or spoken communications, discussions or conversations with teachers. Qualitative method was used to gather information from the different categories of participants. In-depth formal interviewing was used as the main data collection method. The researcher used focus group interviews and individual interviews to collect data. The researcher then interpreted and drew meaning from the displayed data. The knowledge this research intended to create will be of significance because teachers play a key role in the process of implementing assessment policies. Teachers had different views on the process of assessment. All teachers interviewed conceded that they received training on the National Protocol on Assessment. However, educators had different views in terms of the workshops they attended. There must be a shift from norm-referenced to criterion-referenced type of assessment, that is, shift from assessing learners by comparing one learner from the others.
\end{abstract}

Keywords: Teacher, evaluation and assessment, critical discourse analysis, perceptions, forms of assessment, criterion referencing, assessment tasks, progression and promotion.

\section{Introduction and Context of the Study}

There have been critics of the South African education policies which condemned the National Department of Education for a curriculum that is said to be irrelevant and uninteresting for the majority of the South African learners. Before 1994 the education system in South Africa contradicted world trends by deliberately choosing to serve education needs of only a section of the South African population. The birth of democracy in 1994 led to the establishment of a new dispensation and that led to a need for the democratisation of the education system. The apartheid education system has disadvantaged the majority of South Africans especially the black communities. The situation in the new democracy demanded serious changes in many spheres of life in South Africa especially in education.

Curriculum 2005 was introduced in 1997 and piloted in selected schools countrywide. Then in 1998, the Department of Education introduced Curriculum 2005 in all schools in South Africa. However, the changes in education had an impact on the attitude, moral and performance of teachers in general as well as assessment in particular. These changes required a paradigm shift on the part of teachers. The introduction of outcomes-based assessment made many demands on both the educators and the education system. Teachers had to change the way they used assess. It is hoped that the study will also inform policy makers about problems experienced by teachers in the implementation of the various methods of assessment. Assessment policy can have a negative impact on teaching and learning if not appropriately implemented.

Drotar (2007:3), states that in order to significant, the research needs to exceed the threshold of current scientific work in a specific area. It was appropriate to research how these assessment policies are being implemented and their processes managed in schools and their intended value in teaching and learning. The knowledge this research intended to create will be of significance because teachers play a key role in the process of implementing assessment policies.

\section{Theoretical Frameworks}

According to Terre Blanch (2007:20), refining a research problem involves identifying a theoretical framework upon which 
to base the research. It is imperative to state the theories that influenced the research problem as well as the research methods that were used. This study was underpinned by a critical discourse analysis theory in which the researcher expressed his comments or judgements based on written or spoken communications, discussions or conversations with educators. It involved an analysis of the merits and demerits of the implementation of assessment policies. It is for these reasons that critical discourse analysis was used as a framework to engage and converse about the research problem and questions of this study. Critical discourse analysis deals with long term analysis of fundamental causes and consequences of issues. Therefore it requires an account of detailed relationships between text, talk, society and culture.

The main research question was as follows:

* How are teachers implementing assessment policies in selected schools in Gauteng Province?

\section{Reseach Design and Methods}

Trochim (2006:1), states that research design provides the glue that holds the research project together and design is used to structure the research, to show all the major parts of the research projects- the samples or groups, measures, treatments or programmes, and methods of assessment- work together to try to address the central research questions. Tompkins (2008:4) further states that researchers can design a study to characterise a single instance of a phenomenon or take an inference about a phenomenon in a population via a sample. The research methodology of this study was qualitatively and inductively based. Qualitative method was used to gather information from the different categories of participants on how assessment methods are implemented. Qualitative research assisted the researcher to investigate how educators implement assessment policies. This method assisted in answering the research questions which are informed by the main research question enabling critical discourse analysis to examine the spoken and written words in detail.

The target group and population of the study comprised of experienced teachers who are teaching Intermediate Phase learners. Only five schools were invited to participate in the study. The researcher interviewed five teachers in each school. The above description of the sample is an indication that a purposive sample was used in this research. According to Soanes and Stevenson (2008:1167), purposive refers to "having or done with a particular purpose ". In this study purposeful sampling was used to select for informants with rich information. The sampling involved schools from different socio-economic backgrounds.

\section{Data Analysis}

According to Michelle (2007:3), qualitative data analysis consists of identifying, coding and categorising patterns found in the data. Bradley (2007:5) declares that once the data have been reviewed and there is a general understanding of the scope and contexts of the key experiences under study, coding provides the analyst with a formal system to organise data, uncovering and documenting additional links within and between concepts and experiences described in the data. Plooy (2007:41) defines data analysis as a process of bringing order and structure to the mass of collected data. Data analysis and interpretation involved the analysis and interpretation of documents related to assessment policies. Data were analysed by selecting, comparing, synthesising and interpreting information to provide explanation.

\section{Research Findings and Discussions}

\subsection{Question: Please give examples of rubrics or marking grids you may have used for assessment.}

Most teachers interviewed mentioned assessment tools such as memos, rubrics and checklists. They all conceded that the Department of Education supplied them with all the tools necessary for assessment.

To establish teachers' requirements for the successful implementation of assessment policies, the following question was asked:

\subsection{Question: What are your requirements for the successful implementation of the new assessment policies?}

All teachers who participated in this study indicated that they request the Department of Education not to introduce many assessment policies which always change.

The next question was asked to determine educators' challenges in the implementation of the new assessment 
policies.

\subsection{Question: What training did you receive in respect of the new assessment policies?}

All teachers interviewed conceded that they received training for the National Protocol on Assessment.

In order to identify the various aspects of the training the teachers received, they were asked to elaborate more on the workshops they attended.

\subsection{Question: Tell me more about the workshops you have attended.}

In responding to the question above, teachers had different views. This is supported by the different views below:

"In most workshops the facilitators gave us activities and guided us on how to go through the activities and we were given opportunities to demonstrate how we were going to implement these policies in our classrooms".

"When we attended the workshops on the National Protocol on Assessment, we were told different things which were confusing. The facilitators were not interpreting the various documents the same way. We were given a lot of papers to fill in. The presentations in the workshops were not appropriate to what we were practically doing in our classes".

All teachers interviewed conceded that they received training on the National Protocol on Assessment. However, educators had different views in terms of the workshops they attended. This was quite evident to the researcher when some teachers stated that in most workshops the facilitators gave them activities and guided them on how to go through the activities and were also given opportunities to demonstrate how they were going to implement these policies in their classrooms. An indication was also made by other teachers that the presentations in the workshops were not appropriate to what they were practically doing in their classes.

5.5 Question: Did you find the training activities relevant to what you were doing in class? If relevant or irrelevant, explain.

Most teachers indicated that the workshops were relevant but it was not easy to implement what they were trained for in real classroom environment. This was emphasised by one teacher when he said, "Yes, but trainings are relevant, but it is totally different from what we do in class. In class there are those who are very slow, and some very intelligent. The training activities are relevant but more challenging to implement in class. When you present in the workshops there are only teachers but when you present in class is a different story. You are faced with a real situation".

The above response was followed by another question to determine teachers' opinions about the knowledge trainers or co-ordinators had of these assessment policies.

\subsection{Question: What is your opinion about the competency level of the trainers and co-ordinators of the training?}

The findings of this study indicated that most teachers viewed the facilitators of the workshops for C2005, RNCS, and NCS as not being competent enough. Judging from the responses, there was general agreement that the facilitators of the workshops for CAPS were much better. This implied that the educators are more comfortable with CAPS than the previous policies on teaching, learning and assessment. However, it should be indicated that the teachers have not yet implemented CAPS but were referring to training but not implementation. The researcher's assumption was that the facilitators of the workshops on CAPS were more prepared and could satisfy the educators' expectations.

\subsection{Question: Tell me more about assessment procedures you are employing.}

In responding to the question above, the teachers mentioned contradicting statements. A few of the verbatim responses are indicated below:

"As assessment is ongoing, for example, comprehension, you assess two things, reading and comprehending. You ask questions and they respond".

"We get all the guidelines from our facilitators. We know exactly what needs to be done. Under assessment plans, the 
parents can also see what is happening at school".

"I assess my learners at least every second week by means of tests and letters to write".

"We assess against learning outcomes".

"I can't elaborate much on that".

The researcher noticed that the teachers had contradicting views about outcomes-based assessment. This was evident when some teachers indicated that they assess reading and comprehension, others indicated that they assess by using tests and letters, while some indicated that they assess learning outcomes. Furthermore, the lack of knowledge of outcomes-based assessment was evident when some teachers could not even explain what they understood by the concept outcomes-based assessment. Based on the responses of the teachers who participated in this study, it was clear that the concept of outcomes-based assessment was not well understood by the teachers. The researcher is therefore, of the opinion that the facilitators could have given the teachers contradicting statements about outcomesbased assessment.

The next question sought to establish teachers' knowledge of the principles of outcomes-based assessment.

\subsection{Question: What are some of the principles of outcomes-based assessment?}

All teachers interviewed indicated that they don't know any principles of outcomes-based assessment.

\subsection{Question: What are some of the assessment methods you commonly use when assessing your learners?}

The findings of lack of knowledge of the various assessment methods were shown when some educators could not indicate a clear understanding of the different assessment methods. Lack of knowledge of the various assessment methods was also evident from the teachers' responses. Some teachers indicated that assessment methods are the building blocks of tasks while others mentioned that assessment methods are questions and answers. However, some of the teachers had knowledge of the various forms of assessment as they mentioned aspects such as assignments, investigations, essays, projects and responses to texts. The data presented by teachers in this study showed that some educators knew about the various assessment methods while others had no idea of the new methods used in assessing learners' achievements of learning outcomes.

\subsection{Question: What do you understand by different forms of assessment?}

All teachers could not indicate a clear understanding of the different forms of assessment. This was confirmed by one teacher who said, "According to policy, it says these different types of assessment are the building blocks of the tasks. Other learners may know how to read but not know how to comprehend".

\subsection{Question: Please name a few departmental guidelines you use when planning assessment.}

The researcher noticed that the Department of Education had supplied the teachers with assessment guidelines. However, when responding to the researcher's questions, the teachers mentioned some documents such as mark sheets and grids which are in fact, assessment tools. The fact that teachers indicated that they have assessment guidelines but they had challenges in implementing them is an indication that they could not interpret them appropriately.

\subsection{Question: Please comment on the school's reporting policy.}

All teachers who were interviewed mentioned that each school has its own policy which is informed by the one supplied by the Department of Education.

The above responses were followed by another question to determine the criteria teachers used for progression of learners from one grade to the other. 


\subsection{Question: Which criteria are used for progressing learners from one grade to another?}

The teachers indicated that the Department of Education has given them guidelines which they follow when progressing learners from one grade to the other. On analysing the assessment guidelines, the researcher noticed that learners in grades 4 to 8 progress with their age cohort. This contradicted with another guideline that indicated that any decision about progression should be based on the evidence of a learner's performance against recorded assessment tasks. Data further indicated that no learner should stay in the same phase for longer than four years. The researcher is of the view that some learners therefore progress from one grade to the other without having achieved learning outcomes.

\subsection{Question: What evidence is there for recording learners' progress?}

The teachers indicated that they keep some recording sheets at school for a long time. In order to ascertain how teachers report learners' achievement of learning outcomes, the following questions were asked:

\subsection{Question: How is reporting of learners' achievement done?}

The teachers mentioned that reporting is done quarterly.

\section{Conclusions and Recommendations}

It was found that some teachers have changed the way they used to assess after the introduction of the new assessment policies, while other teachers did not change. Schools in urban areas were adequately supplied with assessment materials than the schools in the townships, informal settlements and on the farms. Teachers received training on the various assessment policies. However some policies were not deemed appropriate to what teachers were actually doing in their classrooms. This study established that teachers have inadequate knowledge of outcomes-based assessment. The Department of Education provided schools with resources on assessment policies. However, schools did not receive the resources equally or equitably. Though the Department of Education provided schools with assessment policies, teachers interpreted them differently. There were problems with regard to time allocation for training workshops and the long distances travelled to the training workshop centres. The researcher also observed that, as a result of the long distances travelled the teachers arrived late for the workshops while others were already tired by the time they reached the training centres.

The researcher recommends that for the teachers to be effective and efficient in their daily assessment processes there should be in-service trainings. These are regarded as those education and training activities engaged in by teachers following their initial professional certification and intended mainly or exclusively to improve their professional knowledge, skills and attitudes in order to teach and assess effectively. The Department of Education should train teachers on how to implement assessment methodologies. Education should make learners self reliant and competent. Schools should be provided with sufficient infrastructure that allows easy access to resources for both teachers and learners. The Department of Education is encouraged to give more financial support to schools in order to provide the basic infrastructure. Teachers should improvise resources by planning and executing activities. Policy makers have to explore professional development from the side of the participating educators in order to clearly understand what would be best for changing their assessment practice. It is therefore recommended that for effective implementation of assessment policies, the Department of Education should provide adequate knowledgeable curriculum staff to do extensive training for both the educators. Teachers' training or professional development should be longer, though it might be less than their initial training. Teachers should collaborate and establish learning area/subject clusters to resolve mutual curricular challenges. Teachers should assess learners' achievement of learning outcomes as unpacked by the various assessment standards.

\section{Limitations of the Study and Further Areas of Research}

This research provides an insight in assessment policies South African primary schools. It is a step further in the investigation of assessment policies. However, the sample of this study was drawn from the teachers of selected primary schools in Gauteng Province only. Therefore, it is not representative of the entire population of teachers in this country. Further studies need to be conducted in other provinces. Its findings might not necessarily apply elsewhere as schooling 
in South Africa vary within districts in a province and vary across districts in the country. The sample presented only one district in the Department of Basic Education in Gauteng Province. Only public schools were the target population in this study. Further research, focusing on the private schools is needed.

Another limitation is that teachers could not be observed over a period of time to establish how they implement these assessment policies. Basically, this study serves as a detailed foundation establishing how assessment policies are implemented in South African primary schools. This study therefore does not purport to be a comprehensive and final examination of how assessment policies are implemented. The sample of this study was drawn from the Intermediate Phase classes only. There is need for a study of the Foundation, Senior and Further Education and Training Phases. Only interviews, observations and documents were used to collect data. Further research, using a combination of these tools and others is needed.

In spite of the limitations and further areas of research mentioned above, this study has achieved its objectives of understanding how assessment policies are implemented in South African primary schools.

\section{References}

Bradley, E.H. et al. 2007. Qualitative Data Analysis for Health Services Research. Health Services Research. Vol.156: 618-626. Duffy, T.M. 2006. Constructivism: Implications for the Design and Delivery of Instruction. Indianapolis. Indiana University. Michelle, B. 2007. Data Analysis Strategies for Qualitative Research- Research Corner. AORN Journal. Vol. 7: 103-115. Plooy, G.M. 2007. Communications Research Techniques, Methods and Implications. Claremont. Juta and Co. LTD. Soanes, C. and Stevenson, A. 2008. Concise Oxford English Dictionary. Oxford. Oxford University Press. Terre Blanche, 2008. Research in Practice: Applied methods for the social sciences. Sandston. Juda Academy. Tompkins, W.G. 2008. Research Designs: Choosing and Fine-tuning a Design for your Study. London. Sage Publications. Trochim, M.K. 2006. Research Methods Knowledge Base. Mason. Atomic Dog Publications. 